\title{
Plant-soil feedbacks and the coexistence of competing plants
}

\author{
Tomás A. Revilla • G. F. (Ciska) Veen • \\ Maarten B. Eppinga • Franz J. Weissing
}

Received: 26 April 2011 / Accepted: 10 May 2012 / Published online: 9 June 2012

(C) The Author(s) 2012. This article is published with open access at Springerlink.com

\begin{abstract}
Plant-soil feedbacks can have important implications for the interactions among plants. Understanding these effects is a major challenge since it is inherently difficult to measure and manipulate highly diverse soil communities. Mathematical models may advance this understanding by
\end{abstract}

Tomás A. Revilla and G. F. (Ciska) Veen contributed equally to this paper.

T. A. Revilla $\cdot$ F. J. Weissing $(\bowtie)$

Theoretical Biology Group, Centre for Ecological and

Evolutionary Studies, University of Groningen,

P.O. Box 11103, 9700 CC Groningen, The Netherlands

e-mail: f.j.weissing@rug.nl

\section{T. A. Revilla}

Station d'Ecologie Experimentale du CNRS a Moulis, USR 2936, Moulis,

09200 Saint-Girons, France

\section{G. F. Veen}

Community and Conservation Ecology Group, Centre for Ecological and Evolutionary Studies, University of Groningen, P.O. Box 11103, 9700 CC Groningen, The Netherlands

\section{G. F. Veen}

Department of Terrestrial Ecology,

Netherlands Institute of Ecology,

P.O. Box 50, 6700 AB Wageningen, The Netherlands

G. F. Veen

Department of Forest Ecology and Management, Swedish University of Agricultural Sciences, 90183 Umeå, Sweden

\section{B. Eppinga}

Department of Innovation and Environmental Sciences, Utrecht University,

P.O. Box 80155, 3508 TC Utrecht, The Netherlands

\section{T. A. Revilla}

Instituto de Zoologia y Ecologia Tropical,

Universidad Central de Venezuela,

Av. Paseo Los Ilustres, Los Chaguaramos, Caracas 1041-A,

Venezuela making the interplay of the various processes affecting plant-soil interaction explicit and by quantifying the relative importance of the factors involved. The aim of this paper is to provide a complete analysis of a pioneering plant-soil feedback model developed by Bever and colleagues ( $\mathrm{J}$ Ecol 85: 561-573, 1997; Ecol Lett 2: 52-62, 1999; New Phytol 157: 465-473, 2003) to fully understand the range of possible impacts of plant-soil feedbacks on plant communities within this framework. We analyze this model by means of a new graphical method that provides a complete classification of the potential effects of soil communities on plant competition. Due to the graphical character of the method, the results are relatively easy to obtain and understand. We show that plant diversity depends crucially on two key parameters that may be viewed as measures of the intensity of plant competition and the direction and strength of plantsoil feedback, respectively. Our analysis provides a formal underpinning of earlier claims that plant-soil feedbacks, especially when they are negative, may enhance the diversity of plant communities. In particular, negative plant-soil feedbacks can enhance the range of plant coexistence by inducing competitive oscillations. However, these oscillations can also destabilize plant coexistence, leading to low population densities and extinctions. In addition, positive feedbacks can allow locally stable forms of plant coexistence by inducing alternative stable states. Our findings highlight that the inclusion of plant-soil interactions may fundamentally alter the predictions on the structure and functioning of above-ground ecosystems. The scenarios presented in this study can be used to formulate hypotheses about the ways soil community effects may influence plant competition that can be tested with empirical studies. This will advance our understanding of the role of plant-soil feedback in ecological communities.

Keywords Graphical analysis · Oscillations · Diversity · Plant community dynamics $\cdot$ Bever model 


\section{Introduction}

Ecologists have long recognized that interactions between plants are mediated by many biotic (e.g., grazing, plant competition, and facilitation) and abiotic factors (e.g., soil texture, nutrient availability, and topography; Harper 1977; Tilman 1988). More recent research has stressed the influence of the soil community on competitive interactions between plants (Callaway et al. 2004; Klironomos 2002) by exerting positive or negative effects on the growth of specific plants (De Deyn et al. 2003; Gange et al. 1993; Klironomos 2003; Olff et al. 2000; van der Heijden et al. 1998a; van der Heijden et al. 2003; van der Putten and van der Stoel 1998; van der Putten et al. 1993). Therefore, plantsoil interactions can potentially be of crucial importance for species composition of plant communities and, more generally, the diversity of terrestrial ecosystems (van der Putten et al. 2001; Wardle et al. 2004).

The interaction between a plant and the soil community, referred to as plant-soil feedback, is a two-step process: the presence of a specific plant changes the composition of the soil community, which in turn alters the growth rate of that specific plant (Bever 2003; Reynolds et al. 2003). Quantifying the effect of soil organisms on plant growth and vice versa is difficult due to the vast below-ground diversity, and the technical problems inherent to measuring and manipulating soil communities (Bever 2003; van der Putten et al. 2009). Therefore, there is a need for mathematical models that can help to generate insight into the potential implications of plant-soil feedbacks on species dynamics and diversity (van der Putten et al. 2009).

Bever and colleagues $(1997,1999,2003)$ developed such a modeling framework. In a 2003 paper, Bever incorporated plant-soil interactions in the classical Lotka-Volterra competition model, an approach that motivated several subsequent modeling studies on similar topics (Bonanomi et al. 2005; Eppinga et al. 2006; Eppstein et al. 2006; Eppstein and Molofsky 2007; Umbanhowar and McCann 2005). Bever (2003) highlighted two implications of plant-soil feedback for the coexistence of two competing plant species: (1) negative plant-soil feedback facilitates plant coexistence and (2) negative plant-soil feedback drives oscillations in plant abundances. These kinds of predictions are appealing to empirical plant-soil ecologists because they can be tested with relatively straightforward experiments (Bever 1994; Bever et al. 1997), without requiring specific knowledge on the composition of soil communities or the effects of individual soil-borne species on plant growth.

Bever only performed a partial analysis of his model, thus leaving out a number of interesting predictions. A more complete analysis of the "Bever model" (i.e., the model developed in Bever 2003) is required to fully understand the impact of plant-soil feedbacks on plant communities in this framework. The first goal of our paper is to develop intuitively appealing methods allowing such an analysis, thereby pointing out various routes by which soil communities can affect plant diversity. A second goal is to refine some of Bever's (2003) conclusions, such as the usefulness of the "feedback parameter $I_{\mathrm{S}}$ " (which was introduced on the basis of an earlier model) in the context of plant competition, and the characterization of the parameter range for which plant-soil feedback drives oscillations in plant abundances. Our third goal is to highlight that oscillations induced by negative feedbacks do not per se enhance plant diversity. In fact, we will show that such feedbacks can actually be detrimental for plant coexistence on a local scale.

We start this paper by introducing the Bever model in "The Bever model" section. In the "Graphical analysis" section, we analyze and predict the outcomes of the model by means of a graphical method integrating plant-soil interactions and plant competition. In the "Implications for plant coexistence" section, we derive the implications of our results for plant species coexistence, emphasizing some new results and scenarios not considered by Bever (2003). Finally, we discuss the implications of our results in the "Discussion" section.

\section{The Bever model}

The "Bever model" (Bever 2003) studies the effect of two soil communities with densities $S_{\mathrm{A}}$ and $S_{\mathrm{B}}$ on the growth of two competing plant species with densities $N_{\mathrm{A}}$ and $N_{\mathrm{B}}$ (Fig. 1). The dynamics of the system are described by the following system of four differential equations:

$$
\frac{d N_{\mathrm{A}}}{d t}=r_{\mathrm{A}} N_{\mathrm{A}}\left(1+\alpha_{\mathrm{A}} S_{\mathrm{A}}+\beta_{\mathrm{A}} S_{\mathrm{B}}-\frac{N_{\mathrm{A}}+c_{\mathrm{B}} N_{\mathrm{B}}}{K_{\mathrm{A}}}\right)
$$

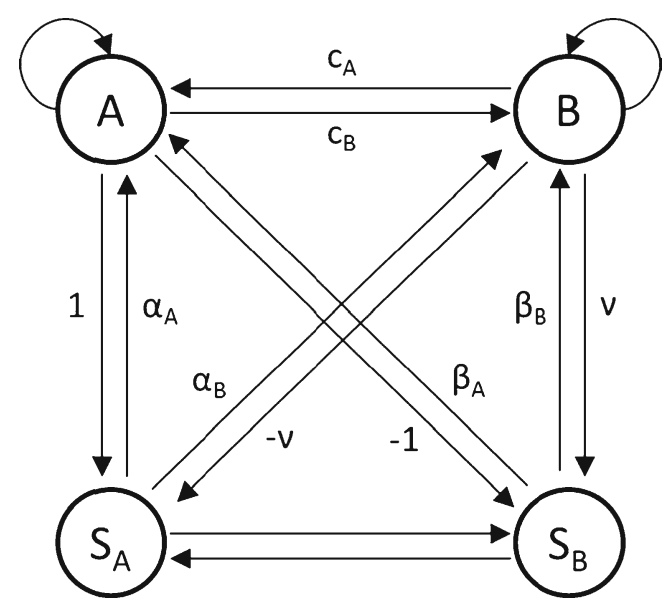

Fig. 1 Schematic representation of the interactions between two plants $\mathrm{A}$ and B and their associated soil communities $S_{\mathrm{A}}$ and $S_{\mathrm{B}}$ 


$$
\frac{d N_{B}}{d t}=r_{B} N_{B}\left(1+\alpha_{B} S_{A}+\beta_{B} S_{B}-\frac{N_{B}+c_{A} N_{A}}{K_{B}}\right)
$$

$\frac{d S_{\mathrm{A}}}{d t}=S_{\mathrm{A}} S_{\mathrm{B}} \frac{N_{\mathrm{A}}-v N_{\mathrm{B}}}{N_{\mathrm{A}}+N_{\mathrm{B}}}$

$\frac{d S_{\mathrm{B}}}{d t}=S_{\mathrm{A}} S_{\mathrm{B}} \frac{v N_{\mathrm{B}}-N_{\mathrm{A}}}{N_{\mathrm{A}}+N_{\mathrm{B}}}$

The competition coefficients $c_{\mathrm{A}}$ and $c_{\mathrm{B}}$ are expressed as the per capita effects of each species on the growth rate of the competitor species (interspecific competition), relative to the per capita effect on the growth rate of its own population (intraspecific competition). $r_{\mathrm{A}}$ and $r_{\mathrm{B}}$ denote the intrinsic per capita growth rates of the plant species. $K_{\mathrm{A}}$ and $K_{\mathrm{B}}$ are the carrying capacities of the plant species when growing in isolation. $S_{\mathrm{A}}$ and $S_{\mathrm{B}}$ are the densities of the two soil communities, where $S_{\mathrm{A}}$ is specifically associated with plant species A and $S_{\mathrm{B}}$ with plant species B. The soil community is positively affected by the relative abundance of its associated plant species and negatively affected by the relative abundance of the other plant species. The parameter $\nu$ is a scaling factor that quantifies the relative strength of the positive and negative effects of plants on soil community growth. The effect of the soil communities on plant growth is characterized by the parameters $\alpha_{\mathrm{A}}, \alpha_{\mathrm{B}}, \beta_{\mathrm{A}}$, and $\beta_{\mathrm{B}}$, respectively, which can be either positive, negative, or zero. The appearance of the product $S_{\mathrm{A}} S_{\mathrm{B}}$ in (1c) and (1d) reflects the fact that Bever originally derived his model for the relative abundances of the two soil communities (Bever et al. 1997). In fact, adding (1c) and (1d) yields $\frac{d S_{\mathrm{A}}}{d t}+\frac{d S_{\mathrm{B}}}{d t}=0$ and, as a consequence, $S_{\mathrm{A}}+S_{\mathrm{B}}=$ const. Scaling the total abundance of the soil communities to one, we can express $S_{\mathrm{B}}$ in terms of $S_{\mathrm{A}}$ (i.e., $S_{\mathrm{B}}=1-S_{\mathrm{A}}$ ) and reduce system (1) to a system of three differential equations. This system can be written in the form:

$\frac{d N_{\mathrm{A}}}{d t}=\rho_{\mathrm{A}} N_{\mathrm{A}}\left(1-\frac{N_{\mathrm{A}}+c_{\mathrm{B}} N_{\mathrm{B}}}{\kappa_{\mathrm{A}}}\right)$

$\frac{d N_{\mathrm{B}}}{d t}=\rho_{\mathrm{B}} N_{\mathrm{B}}\left(1-\frac{N_{\mathrm{B}}+c_{\mathrm{A}} N_{\mathrm{A}}}{\kappa_{\mathrm{B}}}\right)$

$\frac{d S_{\mathrm{A}}}{d t}=S_{\mathrm{A}}\left(1-S_{\mathrm{A}}\right)\left(\frac{N_{\mathrm{A}}-v N_{\mathrm{B}}}{N_{\mathrm{A}}+N_{\mathrm{B}}}\right)$

The intrinsic growth rates $\rho_{A}$ and $\rho_{B}$ and the carrying capacities $\kappa_{A}$ and $\kappa_{B}$ of the two plant species depend on the densities of the soil communities, $S_{\mathrm{A}}$ and $S_{\mathrm{B}}=1-S_{\mathrm{A}}$, and they are given by:

$\rho_{\mathrm{A}}=\rho_{\mathrm{A}}\left(S_{\mathrm{A}}\right)=r_{\mathrm{A}} \cdot\left(1+\alpha_{\mathrm{A}} S_{\mathrm{A}}+\beta_{\mathrm{A}}\left(1-S_{\mathrm{A}}\right)\right)$

$\rho_{\mathrm{B}}=\rho_{\mathrm{B}}\left(S_{\mathrm{A}}\right)=r_{\mathrm{B}} \cdot\left(1+\alpha_{\mathrm{B}} S_{\mathrm{A}}+\beta_{\mathrm{B}}\left(1-S_{\mathrm{A}}\right)\right)$

$\kappa_{\mathrm{A}}=\kappa_{\mathrm{A}}\left(S_{\mathrm{A}}\right)=\kappa_{\mathrm{A}} \cdot\left(1+\alpha_{\mathrm{A}} S_{\mathrm{A}}+\beta_{\mathrm{A}}\left(1-S_{\mathrm{A}}\right)\right)$

$\kappa_{\mathrm{B}}=\kappa_{\mathrm{B}}\left(S_{\mathrm{A}}\right)=\kappa_{\mathrm{B}} \cdot\left(1+\alpha_{\mathrm{B}} S_{\mathrm{A}}+\beta_{\mathrm{B}}\left(1-S_{\mathrm{A}}\right)\right)$

For the rest of this paper, we assume that $\alpha_{\mathrm{A}}, \beta_{\mathrm{A}}, \alpha_{\mathrm{B}}$, $\beta_{\mathrm{B}}>-1$, thus preventing $\kappa_{\mathrm{A}}$ and $\kappa_{\mathrm{B}}$ from turning into negative equilibrium densities. All other model parameters are assumed to be positive.

\section{Graphical analysis}

We analyze the Bever model in four steps. The first three steps do not show new results, but introduce concepts and a line of reasoning that is crucial for understanding our graphical methods in the final step. First, we discuss the effects of the soil dynamics on plants growing in monoculture, which will lead us to the concept of soil stability. Second, we consider the effects of a fixed soil composition on plant competition, which helps us to define the concept of competitive stability. Third, we take into account the net effects of the plant-soil feedbacks, and how to distinguish whether they are positive or negative. Fourth, we integrate our criteria for soil stability, competitive stability, and feedbacks in a graphical method for the analysis of the complete system (2).

Plant monocultures

A monoculture of plant $\mathrm{A}$ is represented by Eqs. (2a, c) with $N_{\mathrm{B}}=0$. This monoculture has two equilibrium states: a first one in which $S_{\mathrm{A}}=1$ and $N_{\mathrm{A}}=\kappa_{\mathrm{A}}(1)=K_{\mathrm{A}}\left(1+\alpha_{\mathrm{A}}\right)$, and a second one in which $S_{\mathrm{A}}=0$ and $N_{\mathrm{A}}=\kappa_{\mathrm{A}}(0)=K_{\mathrm{A}}\left(1+\beta_{\mathrm{A}}\right)$. When $0<S_{\mathrm{A}}<1$, Eq. (2c) implies that $S_{\mathrm{A}}$ converges to 1 while $S_{\mathrm{B}}$ converges to 0 , i.e., the soil community associated with plant A completely eliminates the soil community associated with plant B. In dynamical terms, the first equilibrium is stable with regards to perturbations in the soil community, and it will be classified as soil-stable. Accordingly, the second equilibrium is unstable with regards to perturbations in the soil community, and it will be classified as soil-unstable. Obviously, the same arguments apply to monocultures of plant species B. For later reference, we give the four monoculture equilibria a name: $\mathbf{A}_{\mathbf{A}}$ : soil-stable monoculture of plant $\mathrm{A}, \mathbf{A}_{\mathbf{B}}$ : soil-unstable monoculture of 
plant $\mathrm{A}, \mathbf{B}_{\mathbf{B}}$ : soil-stable monoculture of plant $\mathrm{B}$, and $\mathbf{B}_{\mathbf{A}}$ : soil-unstable monoculture of plant B.

Effect of a static soil community on plant competition

When a soil community remains static, i.e., at constant density, the coefficients $\rho_{\mathrm{A}}, \rho_{\mathrm{B}}, \kappa_{\mathrm{A}}$, and $\kappa_{\mathrm{B}}$ are constant and plant competition is described by a standard LotkaVolterra model. The dynamics of this model is well-known (e.g., Case 2000): the outcome of competition depends on the ability of each species to invade the monoculture of the other species when this species is at its carrying capacity $\left(\kappa_{\mathrm{A}}\right.$ or $\kappa_{\mathrm{B}}$, respectively). From Eq. (2a), it follows that species A can invade the monoculture of species $\mathrm{B}$ if $c_{\mathrm{B}} \kappa_{\mathrm{B}}<\kappa_{\mathrm{A}}$, implying $c_{\mathrm{B}}<\kappa_{\mathrm{A}} / \kappa_{\mathrm{B}}$. Similarly, species $\mathrm{B}$ can invade in the monoculture of species $\mathrm{A}$ if $c_{\mathrm{A}}<\kappa_{\mathrm{B}} / \kappa_{\mathrm{A}}$. This leads to four possible outcomes of competition (Case 2000). If species A (resp. species B) is the only species able to invade the monoculture of the other species, species A (resp. species B) will win the competition. If both species are able to invade the monoculture of the other species, both species will stably coexist. If neither species is able to invade the monoculture of the other species, one of the species will win the competition, the winner depending on initial conditions.

A community equilibrium exists if either both species or none of the species can invade the monoculture of the other species, i.e., if $c_{\mathrm{A}}-\kappa_{\mathrm{B}} / \kappa_{\mathrm{A}}$ and $c_{\mathrm{B}}-\kappa_{\mathrm{A}} / \kappa_{\mathrm{B}}$ have the same sign. The community equilibrium is stable if the two species can mutually invade each other. Multiplying both sides of the invasion criteria $c_{\mathrm{A}}<\kappa_{\mathrm{B}} / \kappa_{\mathrm{A}}$ and $c_{\mathrm{B}}<\kappa_{\mathrm{A}} / \kappa_{\mathrm{B}}$ yields the stability condition $c_{\mathrm{A}} c_{\mathrm{B}}<1$, which is often interpreted as "interspecific competition is on average weaker than intraspecific competition." Summarizing:

existence of community equilibrium :

$$
\left(c_{\mathrm{A}}-\kappa_{\mathrm{B}} / \kappa_{\mathrm{A}}\right)\left(c_{\mathrm{B}}-\kappa_{\mathrm{A}} / \kappa_{\mathrm{B}}\right)>0
$$

$$
\text { stability of community equilibrium : } c_{\mathrm{A}} c_{\mathrm{B}}<1
$$

Figure 2 illustrates the conditions for equilibrium and stability in a plot where the co-ordinate axes correspond to the ratios of (soil dependent) carrying capacities: $x=\kappa_{\mathrm{B}} / \kappa_{\mathrm{A}}$ and $y=\kappa_{\mathrm{A}} / \kappa_{\mathrm{B}}$ (this approach is similar in spirit as the "recovery plane" analysis of Eppinga et al. (2006) where $\kappa_{\mathrm{B}} / \kappa_{\mathrm{A}}-c_{\mathrm{A}}$ and $\kappa_{\mathrm{A}} / \kappa_{\mathrm{B}}-c_{\mathrm{B}}$ are plotted against each other). The relation between $x$ and $c_{\mathrm{A}}$ determines whether $\mathrm{B}$ can invade the monoculture of $\mathrm{A}$, while the relation between $y$ and $c_{\mathrm{B}}$ determines whether $\mathrm{A}$ can invade the monoculture of $\mathrm{B}$. The parameters $x$ and $y$ are not independent but constrained by $x y=\left(\kappa_{\mathrm{B}} / \kappa_{\mathrm{A}}\right)$ $\left(\kappa_{\mathrm{A}} / \kappa_{\mathrm{B}}\right)=1$. In other words, all parameter combinations describing a competitive plant system actually lie on the hyperbola $x y=1$. If $c_{\mathrm{A}} c_{\mathrm{B}}<1$ (Fig. 2a), this hyperbola intersects the coexistence region III, which means that stable coexistence is possible for certain values of $\kappa_{\mathrm{B}} / \kappa_{\mathrm{A}}$. In the case of $c_{\mathrm{A}} c_{\mathrm{B}}>1$ (Fig. 2b), the hyperbola intersects the mutual exclusion region IV and stable coexistence is not possible at all.

For future reference, we will use the term competitive stability to refer to systems allowing a stable plant community equilibrium $\left(c_{\mathrm{A}} c_{\mathrm{B}}<1\right)$ and the term competitive instability for systems where only monoculture equilibria can be stable $\left(c_{\mathrm{A}} c_{\mathrm{B}}>1\right)$. As we shall see later on, the soil community effects (indirectly) affect the balance between intra- and interspecific plant competition among the plant species. Therefore, such soil community effects have the ability to shift the plant system from one competitive regime to another.

Positive and negative plant-soil feedback

Given a pair of values of $\kappa_{\mathrm{A}}$ and $\kappa_{\mathrm{B}}$ determined by $S_{\mathrm{A}}$ (Eqs. $3 \mathrm{c}, \mathrm{d}$ ) the corresponding competitive system can be represented as a point on the hyperbola $x y=1$ in Fig. 2. Since $S_{\mathrm{A}}$ can vary from 0 to 1 , all feasible competitive systems can be mapped as a continuous set of points, i.e., a feasibility arc. The two endpoints of the feasibility arc have the coordinates:

$$
\begin{gathered}
S_{\mathrm{A}}=1: x=x_{\mathrm{A}}=\frac{\kappa_{\mathrm{B}}(1)}{\kappa_{\mathrm{A}}(1)}=\frac{\kappa_{\mathrm{B}}\left(1+\alpha_{\mathrm{A}}\right)}{\kappa_{\mathrm{A}}\left(1+\alpha_{\mathrm{B}}\right)}, \\
y=y_{\mathrm{A}}=\frac{1}{x_{\mathrm{A}}}=\frac{\kappa_{\mathrm{A}}\left(1+\alpha_{\mathrm{A}}\right)}{\kappa_{\mathrm{B}}\left(1+\alpha_{\mathrm{B}}\right)} \\
S_{\mathrm{A}}=0: x=x_{\mathrm{B}}=\frac{\kappa_{\mathrm{B}}(0)}{\kappa_{\mathrm{A}}(0)}=\frac{\kappa_{\mathrm{B}}\left(1+\beta_{\mathrm{B}}\right)}{\kappa_{\mathrm{A}}\left(1+\beta_{\mathrm{A}}\right)}, \\
y=y_{\mathrm{B}}=\frac{1}{x_{\mathrm{B}}}=\frac{\kappa_{\mathrm{A}}\left(1+\beta_{\mathrm{A}}\right)}{\kappa_{\mathrm{B}}\left(1+\beta_{\mathrm{B}}\right)}
\end{gathered}
$$

Figure 3 pictures the feasibility arc as an arrow with its head at $S_{A}=1$ (Eq. 5a) and its tail at $S_{A}=0$ (Eq. 5b). With respect to the $y$-axis the arc can have an upward or a downward orientation. If the orientation is upwards (Fig. 3a), large values of $S_{\mathrm{A}}$ correspond to systems close to (or in) parameter regime I of Fig. 2a, where plant species A is competitively dominant. Small values of $S_{\mathrm{A}}$ correspond to systems close to (or in) regime II where plant B is dominant. In this situation there is a positive plant-soil feedback in the sense that the dominance of one type of soil community $\left(S_{\mathrm{A}}\right.$ or $\left.S_{\mathrm{B}}\right)$ favors the competitive dominance of the associated plant species (A or B). Similarly, there is negative plant-soil feedback if the arc points downwards (Fig. 3b) because the dominance of one type of soil community favors the competitive dominance of the plant species not associated with the dominant soil community. 
(a)

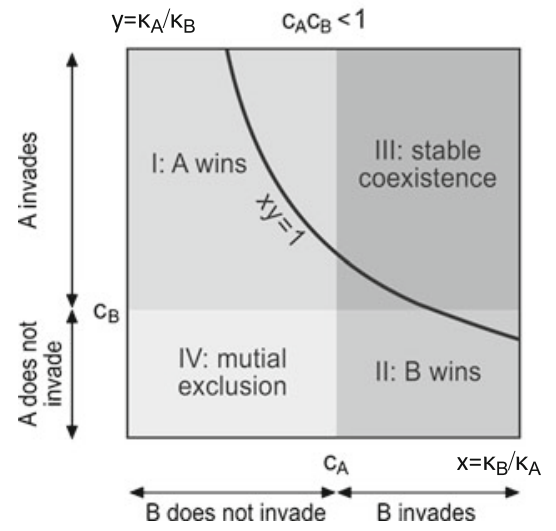

Fig. 2 Outcomes of the Lotka-Volterra system. The axes correspond to the ratios of the carrying capacities: $x=\kappa_{B} / \kappa_{A}, y=\kappa_{A} / \kappa_{B}$. Since $y=1 / x$, all feasible systems are constrained to the hyperbola $x y=1$. Plant $\mathrm{B}$ invades the monoculture of plant $\mathrm{B}$ if $x>c_{A}$ and plant $\mathrm{A}$ invades the monoculture of $\mathrm{B}$ if $y>c_{B}$. As a result the plane can be divided into four invasibility zones I, II, III, and IV. a When $c_{\mathrm{A}} c_{\mathrm{B}}<1$, the hyperbola $x y=1$

The feedback is positive if $y\left(S_{\mathrm{A}}\right)=\kappa_{\mathrm{A}}\left(S_{\mathrm{A}}\right) / \kappa_{\mathrm{B}}\left(S_{\mathrm{A}}\right)$ is increasing with $S_{\mathrm{A}}$ (as in Fig. 3a) or, in other words, if $d y / d S_{\mathrm{A}}>0$. This derivative is given by $d y / d S_{\mathrm{A}}=$ $\left(K_{\mathrm{A}} K_{\mathrm{B}} / \kappa_{\mathrm{B}}^{2}\right) \cdot J_{\mathrm{S}}$, where

$J_{\mathrm{S}}=\left(1+\alpha_{\mathrm{A}}\right)\left(1+\beta_{\mathrm{B}}\right)-\left(1+\alpha_{\mathrm{B}}\right)\left(1+\beta_{\mathrm{A}}\right)$

Since $K_{A} K_{B} / K_{B}^{2}$ is always positive, the plant-soil feedback is positive if $J_{\mathrm{s}}>0$ and it is negative if $J_{\mathrm{s}}<0$. In the absence of plant-soil feedback $\left(J_{\mathrm{s}}=0\right)$, the feasibility arc collapses into a single point (see the "Effect of a static soil community on plant competition" section).

The importance of the sign of the plant-soil feedback was pointed out by Bever and colleagues (Bever et al. 1997;

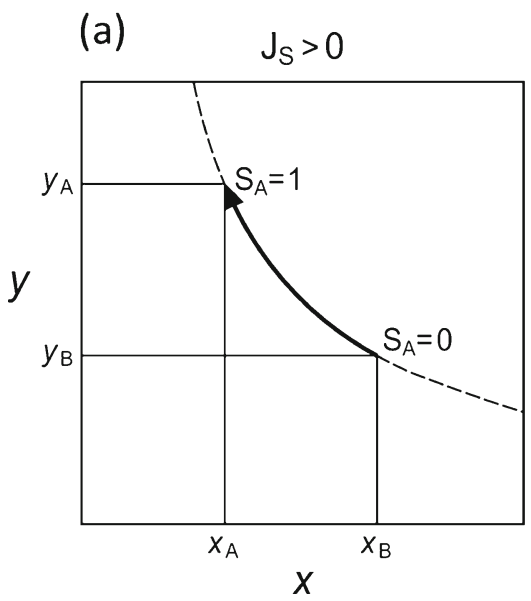

Fig. 3 Effect of positive versus negative plant-soil feedback. The range of possible soil communities ( $S_{\mathrm{A}}$ ranging from 0 to 1 ) determines a feasibility arc, corresponding to a segment on the hyperbola $x y=1$. The direction of increasing $S_{\mathrm{A}}$ is indicated by an arrow head. a If the arrow points upwards $\left(J_{S}>0\right)$, increasing $S_{\mathrm{A}}$ shifts the system towards region I where plant species A wins the (b)

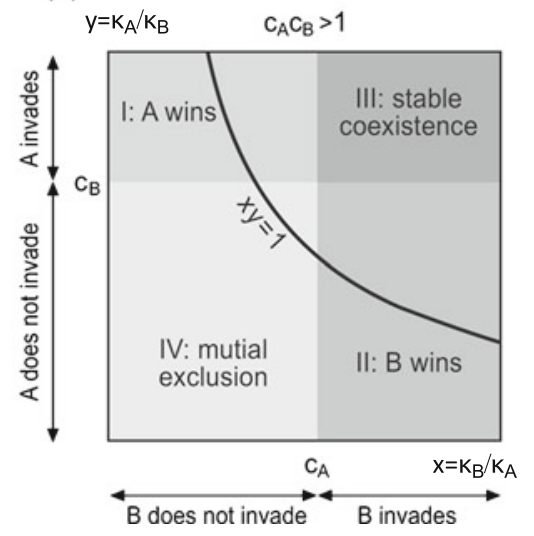

intersects region III, but not region IV. Hence, the community equilibrium is stable (if it exists). b When $c_{\mathrm{A}} c_{\mathrm{B}}>1$, the hyperbola $x y=1$ intersects region IV but not region III. Now the community equilibrium is unstable (if it exists), and depending on the initial conditions, the system will either converge to a monoculture of plant $\mathrm{A}$ or to a monoculture of plant $\mathrm{B}$

Bever 1999) who argued that positive feedback tends to favor competitive dominance and, hence, species-poor plant communities, while negative feedback tends to favor plant coexistence. To quantify plant-soil feedbacks, Bever introduced an interaction coefficient $I_{\mathrm{S}}$ that is defined by

$I_{\mathrm{S}}=\alpha_{\mathrm{A}}+\beta_{\mathrm{B}}-\alpha_{\mathrm{B}}-\beta_{\mathrm{A}}$

There is a simple relationship between the coefficient $I_{\mathrm{S}}$ introduced by Bever, and the coefficient $J_{\mathrm{S}}$ resulting from our analysis

$J_{\mathrm{S}}=I_{\mathrm{S}}+\alpha_{\mathrm{A}} \beta_{\mathrm{B}}-\alpha_{\mathrm{B}} \beta_{\mathrm{A}}$

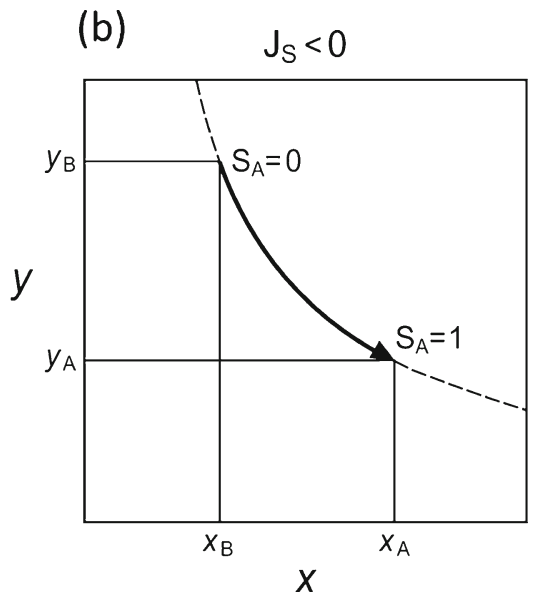

competition (see Fig. 2). Hence, the soil community of A has a positive effect on the competitive position of A (positive soilplant feedback). b If the arrow points downwards $\left(J_{\mathrm{S}}<0\right)$, increasing $S_{\mathrm{A}}$ shifts the system towards region II where species B wins. Hence, the soil community of $\mathrm{A}$ has a negative effect on $\mathrm{A}$ (negative soil-plant feedback) 
Hence, in the case of parameter symmetry (i.e., $\alpha_{\mathrm{A}} \beta_{\mathrm{B}}=$ $\alpha_{\mathrm{B}} \beta_{\mathrm{A}}$ ) Bever's interaction coefficient $I_{\mathrm{S}}$ correctly predicts plant-soil feedback also in the case of plant-plant competition. $J_{\mathrm{S}}$ generalizes $I_{\mathrm{S}}$ to systems without parameter symmetry. In asymmetric scenarios, the difference between $J_{\mathrm{S}}$ and $I_{\mathrm{S}}$ is often small. But it is easy to construct examples where $J_{\mathrm{S}}$ and $I_{\mathrm{S}}$ differ in sign, that is, where Bever's coefficient $I_{\mathrm{S}}$ does not correctly indicate the sign of the feedback.

In analogy to Lotka-Volterra competition coefficients we also define a net plant-soil feedback effect, which can be derived from the ratio between effects on the host plant (analogous to intraspecific competition) and cross-effects on the other plant (analogous to interspecific competition):

$H_{\mathrm{S}}=x_{\mathrm{B}} y_{\mathrm{A}}=\frac{\left(1+\alpha_{\mathrm{A}}\right)\left(1+\beta_{\mathrm{B}}\right)}{\left(1+\alpha_{\mathrm{B}}\right)\left(1+\beta_{\mathrm{A}}\right)}$

In view of (6), $H_{\mathrm{S}}>1$ implies that host-plant effects of a soil community are more favorable than cross-effects, meaning that plant-soil feedback is positive $\left(J_{\mathrm{S}}>0\right) . H_{\mathrm{S}}<1$ implies that cross-effects are more favorable than host plant effects, meaning that plant-soil feedback is negative. We also want to remark that the natural logarithm of $H_{\mathrm{S}}$, which has the literal signs ( + or - ), as well as comparable scales for positive and negative feedbacks, can be approximated by Bever's $I_{\mathrm{S}}$ for small values of alphas en betas (i.e., first order Taylor series).

\section{Combining plant and soil dynamics}

Combining the plots in Figs. 2 and 3 provides us with a graphical method that is often sufficient for a complete characterization of the dynamics of the coupled plant-soil community described by Eq. (2). There are 20 different ways in which the feasibility arc can intersect the four parameter domains corresponding to the plant competition scenarios I to IV (Fig. 4), which are characterized by the monoculture invasion criteria. A detailed overview of the scenarios is given in Appendix A.

Figure 5a depicts case 12, where the feasibility arc lies completely within region II where only species B can invade. We can therefore conclude that irrespective of the state (a)
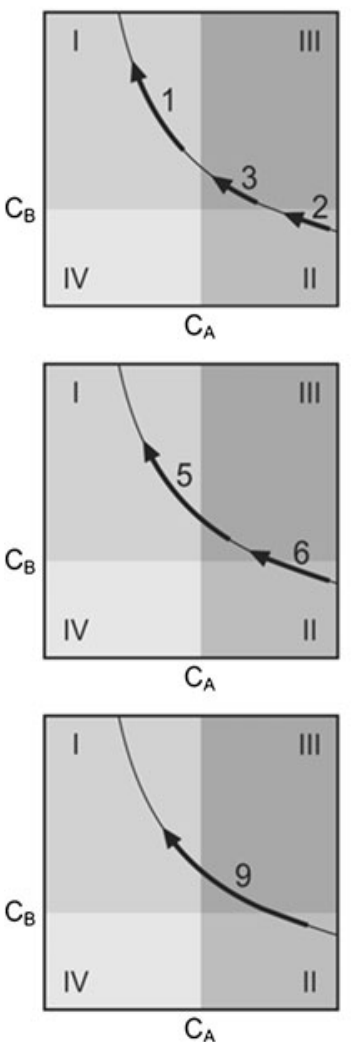

net positive feedback
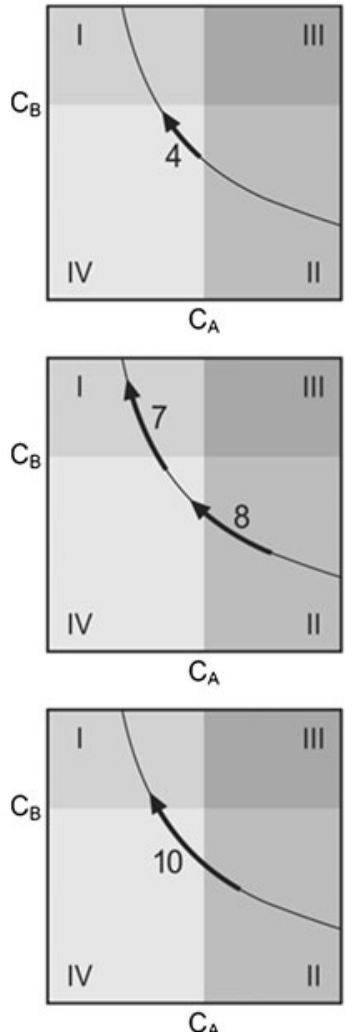

(b)
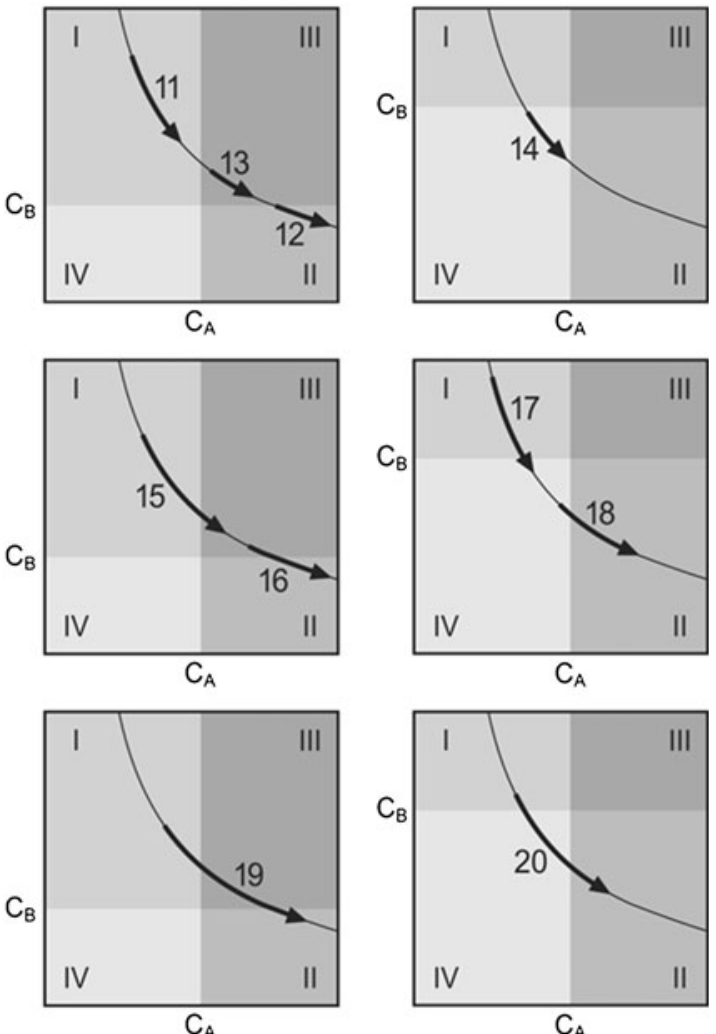

Fig. 4 Intersection of the feasibility arc of Fig. 3 with the invasion zones of Fig. 2. The arc is represented as an arrow which indicates the direction of increasing $S_{\mathrm{A}}$. There are 20 scenarios, differing in the relative position and orientation of the feasibility arc with respect to the invasion zones 
(a)

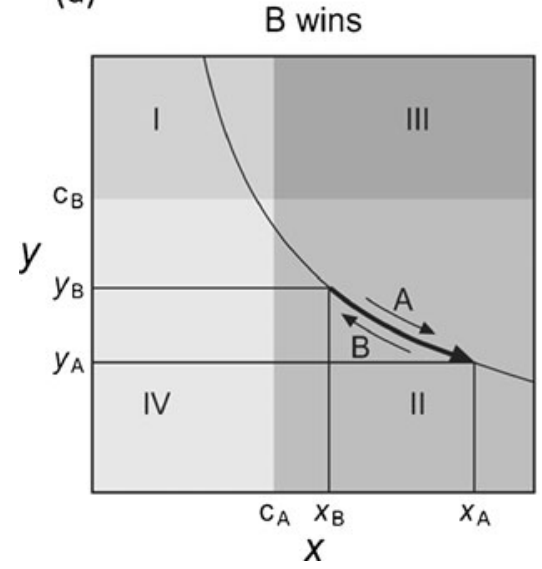

Fig. 5 Graphical analysis of two scenarios discussed in the text. a Case 12 in Fig. 1: the whole feasibility arc is lying in region II. In this case, species B will win the competition, and the soil composition will converge to $S_{\mathrm{A}}=0$ (i.e., the "tail" of the arrow). b Case 15 in Fig. 1: the tail of

of the soil only plant species B can grow in this scenario. Thus the system converges to the monoculture equilibrium $\mathbf{B}_{\mathbf{B}}$ in which $S_{\mathrm{A}}=0$. In fact, in all cases where the feasibility arc lies within regions I or II (cases 1, 2, 11, and 12) one of the plants will always win, irrespective of the initial conditions. When the arc lies in region III (cases 3 and 13), each plant species can invade the monoculture of the other species, implying that the two species will stably coexist at equilibrium. When the arc lies within region IV (cases 4 and 14) neither plant species can invade when rare. Both plant monocultures are stable, and the winner depends on the initial conditions (founder control; Bolker et al. 2003). Summarizing, we can conclude that in the eight scenarios where the feasibility arc lays completely inside one of the four competition regions the qualitative outcome of plant competition does not depend on the sign of the plantsoil feedback.

Figure $5 \mathrm{~b}$ depicts case 15 , which is more complex because the feasibility arc spans two invasion zones. However, the analysis is still straightforward. A monoculture of plant B can never be stable, since the whole arc lies in the parameter region where A can invade such a monoculture. In parameter regime $\mathrm{I}$, a monoculture of plant $\mathrm{A}$ is to be expected in the absence of plant-soil feedback. However, as long as plant A is in monoculture, the associated soil community $S_{\mathrm{A}}$ will increase. Hence the system will be shifted along the feasibility arc in the direction of the arrow, until the plant coexistence regime III is reached. We conclude that in this case the (negative) plant-soil feedback enables coexistence of the two plant species.

As indicated by Fig. 5, the position of the end point of the feasibility arc with respect to the invasion zones tells us whether a plant monoculture can be invaded or not, and (b)

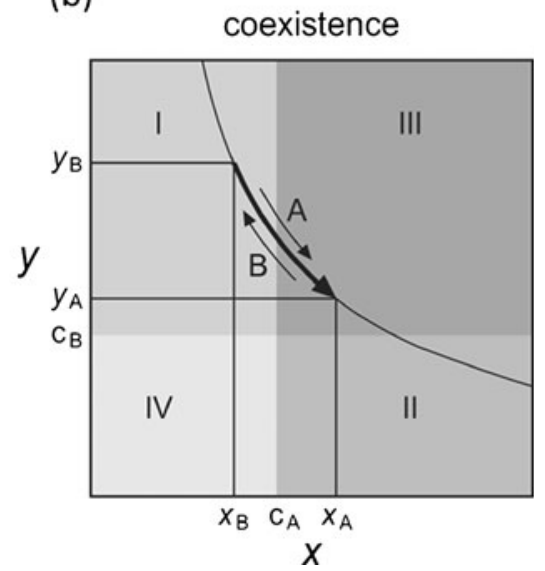

the arc is in region I, whereas the head is in region II. As long as the system is in region I, $S_{\mathrm{A}}$ will increase. Eventually region III will be reached, corresponding to the stable coexistence of the two plant species

hence how the plant-soil interactions may determine the plant dynamics. For a monoculture of plant A, the "head" of the feasibility arc $\left(S_{\mathrm{A}}=1\right)$, which corresponds to the equilibrium state $\mathbf{A}_{\mathbf{A}}$, is relevant. This state can be invaded by plant $\mathrm{B}$ if and only if $c_{\mathrm{A}}<x_{\mathrm{A}}$. By symmetry, a monoculture of plant $\mathrm{B}$ corresponds to the state $\mathbf{B}_{\mathbf{B}}$, which is located at the "tail" of the feasibility arc $\left(S_{\mathrm{A}}=0\right)$ and can be invaded by plant $\mathrm{A}$, if and only if $c_{\mathrm{B}}<x_{\mathrm{B}}$. Multiplying these two inequalities and noticing that $x_{\mathrm{A}} y_{\mathrm{B}}=\left(x_{\mathrm{B}} y_{\mathrm{A}}\right)^{-1}=H_{\mathrm{S}}^{-1}$, we obtain a necessary condition for mutual invasion of the two monoculture equilibria:

$c_{\mathrm{A}} c_{\mathrm{B}}<H_{\mathrm{S}}^{-1}$

In the absence of plant-soil feedback $\left(J_{\mathrm{S}}=0\right.$ or $\left.H_{\mathrm{S}}=1\right)$ condition (10) turns into condition (4b) for the stable coexistence of the two plant species in the Lotka-Volterra competition model. In line with the predictions of Bever and colleagues (Bever 1999; Bever et al. 1997), condition (10) leads to the conclusion that the conditions for plant coexistence are more stringent in the case of a positive feedback (where $H_{\mathrm{S}}^{-1}<1$ ), while they are more relaxed in the case of a negative plant-soil feedback.

Indeed we found that positive feedback generally enhances plant monocultures (cases 7-10), while net negative feedback enhances plant coexistence (cases 15, 16, 19, and 20). However, we found a few exceptions where plants can coexist under positive feedback (cases 5 and 6 ), and where negative feedback drove the system towards plant monocultures (cases 17 and 18). Therefore, in contrast to standard Lotka-Volterra theory, monoculture invasion conditions as those considered above do no longer provide a complete picture of the outcome of plant competition. In the "Implications for plant coexistence" 
section, we will demonstrate in more detail that the two plant species can stably coexist even in cases where condition (10) for the mutual invasibility of the two plant monocultures is not satisfied.

\section{Implications for plant coexistence}

Can plant-soil feedback drive community oscillations?

One of the most important conclusions from Bever (2003), resulting from a numerical example, is that negative plantsoil feedback can enhance coexistence by inducing competitive oscillations. However, a mathematical analysis of the same example (Revilla 2009) reveals that these oscillations rapidly dampen out and that the system converges to a stable coexistence equilibrium. (Only, when numerically integrating the model with a large integration step size we obtained the oscillations described in Bever's Fig. 1c.). Therefore, we first explore if and under what conditions oscillation can arise in the Bever model.

Population cycles are frequently associated with predator-prey dynamics, but they are also a common feature in Lotka-Volterra competition models (Gilpin 1975; May and Leonard 1975) and in resource competition models (Huisman and Weissing 2001; Revilla and Weissing 2008). In these models, oscillations require at least three competitors such that species $\mathbf{R}$ outcompetes species $\mathbf{S}$, $\mathbf{S}$ outcompetes $\mathbf{P}$, and $\mathbf{P}$ outcompetes $\mathbf{R}$, as in the Rock-ScissorsPaper game. Mathematically, the monocultures of $\mathbf{R}, \mathbf{P}$, and $\mathbf{S}$ are connected by heteroclinic orbits, i.e., a sequence of paths
$\mathrm{R} \rightarrow \mathrm{P} \rightarrow \mathrm{S} \rightarrow \mathrm{R}$ that forms a cycle. Although the Bever model involves only two plant competitors, it has in fact four monoculture states $\left(\mathbf{A}_{\mathbf{A}}, \mathbf{A}_{\mathbf{B}}, \mathbf{B}_{\mathbf{B}}, \mathbf{B}_{\mathbf{A}}\right)$. This allows us to construct a heteroclinic cycle as follows.

Consider the configuration corresponding to case 20 in Fig. 6a. From the positioning of the end points of the feasibility arc in competition regimes I and II, we can conclude that the following inequalities are satisfied:

$x_{\mathrm{B}}<c_{\mathrm{A}}<x_{\mathrm{A}}$ and $\mathrm{y}_{\mathrm{A}}<\mathrm{c}_{\mathrm{B}}<\mathrm{y}_{\mathrm{B}}$

As discussed above, the inequalities $c_{\mathrm{A}}<x_{\mathrm{A}}$ and $c_{\mathrm{B}}<y_{\mathrm{B}}$ imply that both plant monocultures can be invaded by their plant competitor when their associated soil communities (i.e., soil community $\mathrm{A}$ is associated to plant monoculture A, and soil community $B$ to plant monoculture B) are dominant. In other words, $\mathbf{A}_{\mathbf{A}}$ and $\mathbf{B}_{\mathbf{B}}$ are competitively unstable. The inequalities $x_{\mathrm{B}}<c_{\mathrm{A}}$ and $y_{\mathrm{A}}<c_{\mathrm{B}}$ imply that the two monocultures cannot be invaded when the invader's soil biota is dominant. In other words, $\mathbf{A}_{\mathbf{B}}$ and $\mathbf{B}_{\mathbf{A}}$ are competitively stable. However, as shown in the "Plant monocultures" section, these two equilibria are not soil stable. In such a situation, we expect a cyclic sequence $\mathbf{A}_{\mathbf{A}} \rightarrow \mathbf{B}_{\mathbf{A}} \rightarrow \mathbf{B}_{\mathbf{B}} \rightarrow$ $\mathbf{A}_{\mathbf{B}} \rightarrow \mathbf{A}_{\mathbf{A}}$ of successions or at least oscillations following this sequence (Fig. 6b).

This is confirmed by Fig. 7 which depicts the time course of the system under the above scenario. For Bever's parameterization (Fig. 7a; corresponding to Bever 2003, Fig. 1c), the system exhibits damped oscillations that converge to a (a)

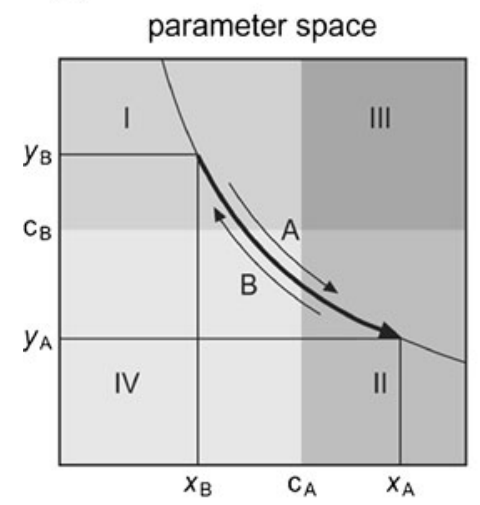

(b)

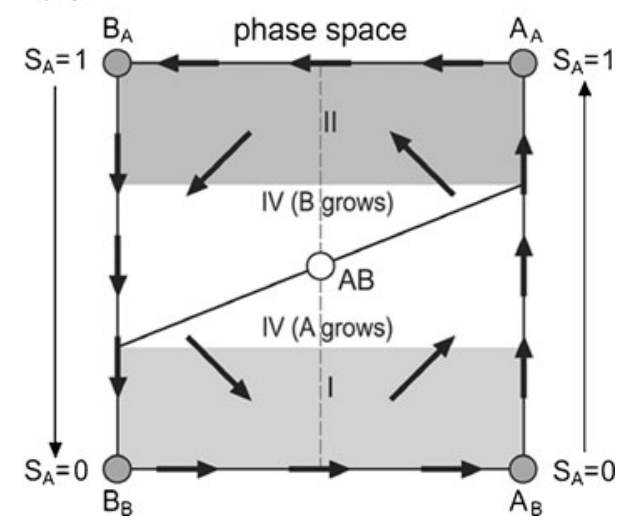

into the phase space). The diagonal line represents the $\mathrm{A}-\mathrm{B}$ nullcline (species A grows towards the right B grows towards the left), and the dashed line is the soil nullcine $\left(S_{\mathrm{A}}\right.$ increases in the right and decreases in the left); their intersection corresponds to the internal equilibrium predicted in (a). Monocultures are either competitively stable or soil stable, but not both: they are saddle points forming a heteroclinic cycle around the internal equilibrium $\mathbf{A B}$ which is competitively unstable. The system converges to a heteroclinic orbit (the border of the phase space) 
(a)
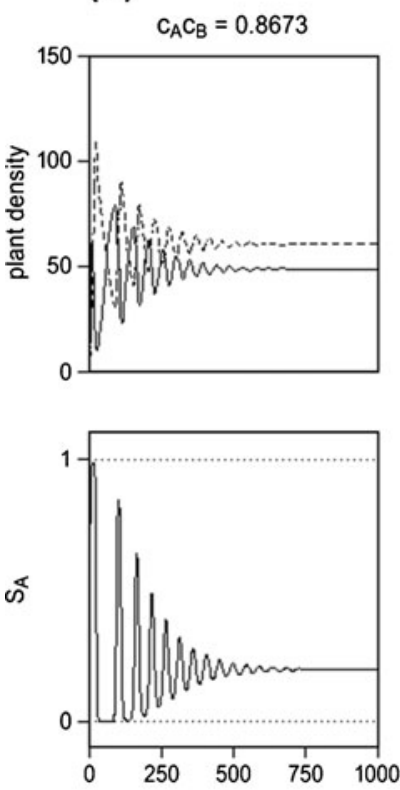

(b)
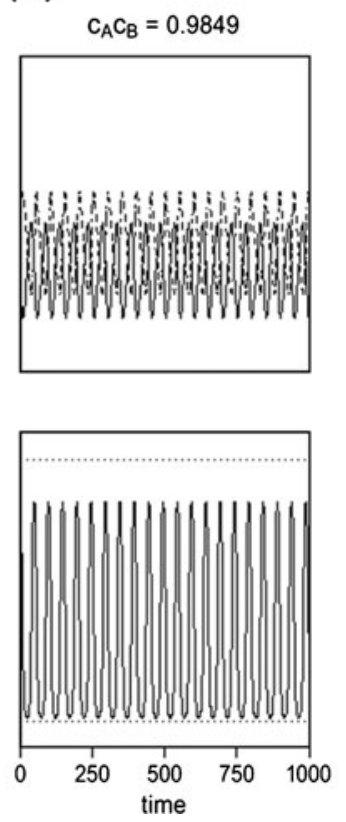

(c)
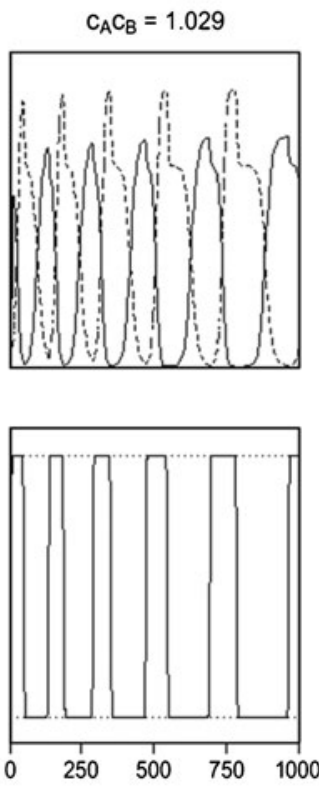

Fig. 7 Competitive oscillations due to a cyclic succession of competitive instability and soil instability. The top panels show the densities of plant A (solid line) and B (dashed line); the bottom panels depict the density of the soil biota $S_{\mathrm{A}}$. The parameter values $K_{\mathrm{A}}=100, K_{\mathrm{B}}=120$, $r_{\mathrm{A}}=0.7, r_{\mathrm{B}}=0.5, \nu=0.8, \alpha_{\mathrm{A}}=-0.03, \alpha_{\mathrm{B}}=\beta_{\mathrm{A}}=0.10, \beta_{\mathrm{B}}=-0.20$, and $c_{\mathrm{B}}=0.980$ are kept fixed, while the competition coefficient $c_{\mathrm{A}}$ is increased from $c_{\mathrm{A}}=0.885$ in (a) via $c_{\mathrm{A}}=1.005$ in (b), to $c_{\mathrm{A}}=1.050$ in (c). Scenario (a) corresponds to Bever's (2003) Fig. 4c with increasing

coexistence equilibrium. By increasing the intensity of competition, $c_{A} c_{B}$, this equilibrium becomes unstable and gives rise to a limit cycle (Fig. 7b). With a further increase in competition intensity the cycle becomes a heteroclinic orbit, i.e., the system "visits" the four monoculture equilibria in a cyclic fashion, remaining in the vicinity of each monoculture state for increasingly longer times (Fig. 7c).

In Fig. $7 \mathrm{a}, \mathrm{b}, c_{\mathrm{A}} c_{\mathrm{B}}<1$ and the parameter configuration corresponds to case 19 in Fig. 1. Now the feasibility arc intersects parameter region III for stable competitive coexistence. In the absence of soil community effects, there exists a stable coexistence equilibrium, but this equilibrium can be destabilized when soil community effects are incorporated. This is indeed the case if the competition intensity $c_{\mathrm{A}} c_{\mathrm{B}}$ is just below 1 (as in Fig. 7b). In fact, it can be shown analytically (Revilla 2009) that stable equilibrium coexistence (as in Fig. 7a) is only possible if $c_{\mathrm{A}} c_{\mathrm{B}}<1-\delta$, where $\delta$ is a positive quantity that can be calculated from the remaining system parameters. In Fig. $7 \mathrm{c}, c_{\mathrm{A}} c_{\mathrm{B}}>1$, as a consequence, the parameter configuration corresponds to case 20 in Fig. 1, where the feasibility arc intersects parameter region IV for mutual competitive exclusion (see also Fig. 6a). In this region, there exists a coexistence intensity of competition, the asymptotic behavior of the system changes from convergence to a stable coexistence equilibrium in (a), via convergence to a limit cycle in (b) to convergence to a heteroclinic orbit in (c). In this example, soil-plant feedback is negative $\left(J_{\mathrm{s}}=-0.434\right)$. Because of $x_{\mathrm{A}} \approx 1.36, y_{\mathrm{A}} \approx 0.73, x_{\mathrm{B}} \approx 0.87, y_{\mathrm{B}} \approx 1.15$, the conditions (13) for the cyclical succession $\mathbf{A}_{\mathbf{A}} \rightarrow \mathbf{B}_{\mathbf{A}} \rightarrow \mathbf{B}_{\mathbf{B}} \rightarrow \mathbf{A}_{\mathbf{B}} \rightarrow \mathbf{A}_{\mathbf{A}}$ are satisfied

equilibrium, but this equilibrium is unstable. As a consequence, the sequence of events described unfolds, and the system converges to the heteroclinic cycle $\mathbf{A}_{\mathbf{A}} \rightarrow \mathbf{B}_{\mathbf{A}} \rightarrow \mathbf{B}_{\mathbf{B}} \rightarrow \mathbf{A}_{\mathbf{B}} \rightarrow \mathbf{A}_{\mathbf{A}}$ (see Fig. 6b).

Notice that the conditions (11) imply that $H_{\mathrm{S}}=x_{\mathrm{B}} y_{\mathrm{A}}<$ $x_{\mathrm{A}} y_{\mathrm{B}}=H_{\mathrm{S}}^{-1}$, which is only possible for $H_{\mathrm{S}}<1$ or, equivalently, $J_{\mathrm{S}}<0$. As a consequence, the above scenario for the development of oscillations can only occur in case of negative plant-soil feedback. Since the analytical results of Revilla (2009) suggest that this is the only route to oscillations in the Bever model, we conclude that competitive oscillations will only occur if two requirements are met: (a) the plant-soil feedback is negative and (b) both soil-stable monoculture equilibria are susceptible to invasion.

Does negative plant-soil feedback enhance coexistence?

Coexistence between plant species is favored when each plant species can invade the monoculture of the other species. This is possible in many scenarios (Fig. 1 scenarios $1,2,3,11,12,13,15,16,19$, and 20). When we exclude the scenarios where feedback does not determine invasion $(1,2,3,11,12$, and 13) we can see 
that only negative plant-soil feedback allows mutual invasion. Although this generally enhances plant coexistence, it does not necessarily lead to equilibrium coexistence. Instead, as shown in the previous paragraph oscillations can occur. If these oscillations take the form of a limit cycle, the two plant species will still stably persist, although not in equilibrium. But the oscillations can also take the form of a heteroclinic cycle, where the populations are repeatedly driven to very low densities. In the mathematical model, they still survive, but in the real world local extinction can occur. Thus, in case of a heteroclinic cycle mutual invasibility of the two monocultures may not result in long-term coexistence.

To assess the relative importance of limit cycles versus heteroclinic cycles, we investigated the pattern in Fig. 7 numerically. Using continuation-bifurcation analysis software (program: XPPAUT, Ermentrout 2002), we found that as $c_{A} c_{B}$ increases, regular oscillations start at $c_{\mathrm{A}} c_{\mathrm{B}}=0.977$ (where a Hopf bifurcation occurs). Already at $c_{\mathrm{A}} c_{\mathrm{B}}=0.998$, these oscillations turn into a heteroclinic orbit. Switching between the four monoculture states persists until $c_{\mathrm{A}} c_{\mathrm{B}}=1.559$ (at this point, the requirement for mutual invasion (12) is no longer satisfied, and the chain of heteroclinic orbits connecting the four monocultures is broken). Thus, while the system does not converge to equilibrium for $0.977<c_{\mathrm{A}} c_{\mathrm{B}}<1.559$, a limit cycle only occurs for the much smaller range $0.977<c_{A} c_{B}<0.998$. In other words, more than $95 \%$ of the parameter range corresponding to non-equilibrium dynamics corresponds to the occurrence of heteroclinic cycles, and hence, to eventual extinction.

Accordingly, negative feedback has a much smaller potential for the facilitation of plant coexistence than Bever's analysis seems to suggest. One should notice, however, that in a spatial context, the risk of extinction via the largeamplitude oscillations associated with a heteroclinic cycle may be counteracted by the repeated re-immigration of the locally extinct species. Under such conditions, also a heteroclinic cycle can allow the regional coexistence of the plant species.
Coexistence as an alternative stable state

The previous example highlights that plant-soil feedbacks can generate competitive dynamics that are not observed in standard competition models. In this section, we will also show an example of how soil community effects can fundamentally alter the stability of equilibria. This has important implications for the utility of invasion criteria to analyze stability. When properly used, invasion criteria are very useful to predict the range of dynamics that a dynamical system can possibly display. In the present context, we can list all possible dynamics of Bever's model (Table 1) by just considering whether $\mathbf{A}_{\mathbf{A}}$ and $\mathbf{B}_{\mathbf{B}}$ can be invaded or not (i.e., whether $c_{\mathrm{A}}<x_{\mathrm{A}}, c_{\mathrm{B}}<y_{\mathrm{B}}$ hold or not), and the direction of the feedback (the sign of $J_{S}$ ). However, as illustrated by the following example an invasion analysis can also have its limitations.

Consider the scenario in Fig. 8a (case 5) where the feedback is positive $\left(J_{\mathrm{S}}>0\right)$ and where $c_{\mathrm{A}}>x_{\mathrm{A}}$ and $c_{\mathrm{B}}<y_{\mathrm{B}}$. In this case, condition (10) for mutual invasibility does not hold: while plant $\mathrm{A}$ is able to invade the monoculture of plant $\mathrm{B}$ (since $c_{\mathrm{B}}<y_{\mathrm{B}}$ ), plant $\mathrm{B}$ cannot invade the monoculture of plant A (since $c_{\mathrm{A}}<x_{\mathrm{A}}$ does not hold). However, stable coexistence may still be possible. As indicated in Fig. 8a, the "tail" of the feasibility arc $\left(S_{\mathrm{A}}=0\right)$ lies in zone III, which means that for $S_{\mathrm{A}}=0$ there is a competitively stable community equilibrium. Following our previous notation, this equilibrium will be denoted by $\mathbf{A B}_{\mathbf{B}}$ since the soil is dominated by B's soil biota, as seen in Fig. 8b. Provided that $N_{\mathrm{A}}<v N_{\mathrm{B}}$, this community equilibrium is a stable attractor: according to Eq. (1c), this condition ensures $d S_{\mathrm{A}} / d t<0$; and hence, the soil stability of $\mathbf{A B}_{\mathbf{B}}$ (in addition to the competitive stability of this equilibrium). Interestingly, $\mathbf{A B}_{\mathbf{B}}$ is not the only attractor: the "head" of the feasibility arc $\left(S_{\mathrm{A}}=1\right)$ lies in zone I, where plant coexistence is not possible and plant $\mathrm{A}$ attains a competitively stable and soil stable monoculture $\mathbf{A}_{\mathbf{A}}$.

This example illustrates three points. First, a locally stable community equilibrium is not necessarily globally

Table 1 Plant community composition for different scenarios of plant invasion and net plant-soil feedback

\begin{tabular}{ccc}
\hline & Net positive feedback $J_{\mathrm{S}}>0$ & Net negative feedback $J_{\mathrm{S}}<0$ \\
\hline $\begin{array}{c}\text { Both plants invade } \\
c_{\mathrm{A}}<X_{\mathrm{A}}, c_{\mathrm{B}}<y_{\mathrm{B}}\end{array}$ & $\begin{array}{c}\text { Stable coexistence with } S_{\mathrm{A}}=1 \text {; stable coexistence with } S_{\mathrm{A}}=0 \text {; stable } \\
\text { coexistence with } S_{\mathrm{A}}=0 \text { or 1, depending on the initial conditions }\end{array}$ & $\begin{array}{c}\text { Stable coexistence with } 0<S_{\mathrm{A}}<1 \text {, competitive } \\
\text { oscillations, limit cycles or heteroclinic cycles }\end{array}$ \\
$\begin{array}{c}\text { A invades } c_{\mathrm{B}}<y_{\mathrm{B}}, \\
\text { B does not } c_{\mathrm{A}}>x_{\mathrm{A}}\end{array}$ & A wins or stable coexistence, depending on the initial conditions & A wins \\
$\begin{array}{c}\text { B invades } c_{\mathrm{A}}<x_{\mathrm{A}} \\
\text { A does not } c_{\mathrm{B}}>y_{\mathrm{B}}\end{array}$ & B wins or stable coexistence, depending on the initial conditions & B wins \\
$\begin{array}{c}\text { None invade } \\
c_{\mathrm{A}}>x_{\mathrm{A}}, c_{\mathrm{B}}>y_{\mathrm{B}}\end{array}$ & Winner depends on the initial conditions & Winner depends on the initial conditions \\
\hline
\end{tabular}


(a)

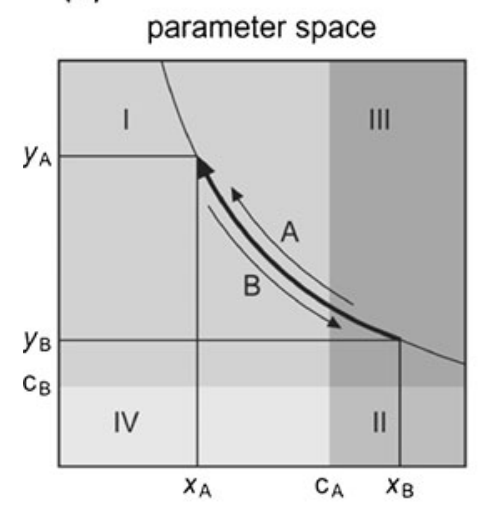

Fig. 8 (a) Graphical analysis and (b) phase space analysis of case 5 in Fig. 1. (a) In this configuration a monoculture of species $A$ is not invasible by B (and hence stable), while a monoculture of species B is invasible by $\mathrm{A}$. As explained in the text, the community equilibrium corresponding to the tail of the feasibility arc can also be a stable equilibrium. Hence, the system allows two alternative stable states. (b) The phase space shows the equilibria (white, unstable; black, stable; gray, saddle) and the invasion zones. The diagonal line representing

stable. In addition to the community equilibrium (here $\mathbf{A B}_{\mathbf{B}}$ ), there can be other stable attractors (here $\mathbf{A}_{\mathbf{A}}$ ), and the competitive outcome depends on the initial conditions. Second, invasion analysis is a very powerful technique, but it has its limitations. In the above example, $\mathbf{A B}_{\mathbf{B}}$ can be stable even in the absence of mutual invasibility (the monoculture equilibrium $\mathbf{A}_{\mathbf{A}}$ cannot be invaded by plant species B). Third, since our graphical analysis reflects an invasion analysis, it also has its limitations. In the above example, the stability of $\mathbf{A B}_{\mathbf{B}}$ depends on the magnitude of the parameter $\nu$, while $\nu$ does not play a significant role in the graphical analysis.

Our conclusion that mutual invasibility is not required for stable plant coexistence is not only of theoretical interest, but also of empirical relevance. In fact, many experimental studies on plant-soil feedback use a set-up that is quite comparable to a mathematical invasion analysis (i.e., introduction of a plant species in a community dominated by other species). The above example demonstrates that - in contrast to classical competition theory-predictions on plant coexistence that are solely based on the outcome of invasion experiments have to be treated with care.

\section{Discussion}

In this paper, we aimed at a better understanding of plant species dynamics and coexistence in the presence of plant-soil feedback. To this end, we developed mathematical and graphical techniques allowing a rather complete analysis of Bever's (2003) model. We found (b)

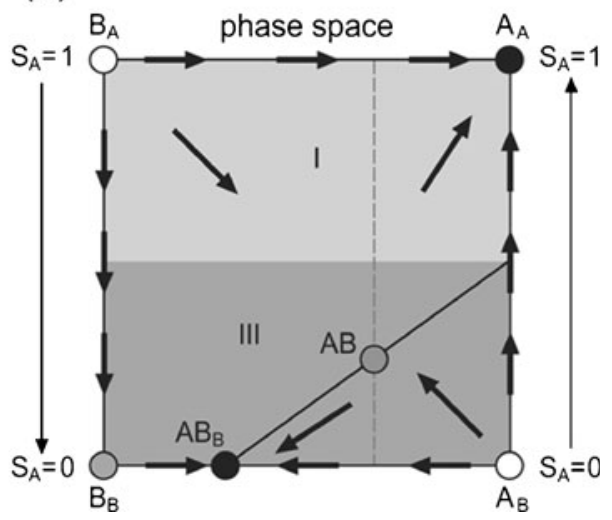

the A-B nullcline (both species grow towards it) intersects $S_{\mathrm{A}}=0$ giving rise to the coexistence equilibrium $\mathbf{A B}_{\mathbf{B}}$ predicted in part (a). If the vertical dashed line representing the soil-nullcline $\left(S_{\mathrm{A}}\right.$ increases to the right and decreases to the left) intersects the A-B nullcline there will be an internal equilibrium $\mathbf{A B}$ that is a saddle point; and depending on the initial conditions the system moves towards the monoculture of $\mathbf{A}_{\mathbf{A}}$ dominated by A's soil biota or towards the coexistence equilibrium $\mathbf{A B}_{\mathbf{B}}$ dominated by B's soil biota

that plant species coexistence is possible if the intensity of interspecies competition $\left(c_{\mathrm{A}} c_{\mathrm{B}}\right)$ is smaller than $H_{\mathrm{S}}^{-1}$, a parameter closely related to the net plant-soil feedback parameter $J_{\mathrm{S}}$. When plant-soil feedback is positive, plant coexistence is more difficult to achieve than in the pure competition model. In contrast, a negative plant-soil feedback allows plant coexistence under more relaxed conditions and thus has the potential to enhance plant community diversity (Bever et al. 1997; van der Heijden et al. 2008). Recent empirical studies, as well as a meta-analysis of more than 300 plant-soil feedback experiments, indeed show that negative feedback favors coexistence and diversity (Bever 1994; Bradley et al. 2008; De Deyn et al. 2003; Kulmatiski et al. 2008; Mills and Bever 1998).

Most of the results from our graphical analysis have an intuitive explanation because plant-soil feedback contributes to the competitive strength of the plant species. For example, in the case of negative plant-soil feedback $\left(J_{\mathrm{S}}>0\right)$, the competitive position of a plant is weakened when its associated soil community increases (i.e., the system shifts towards the region where the other plant species wins, Figs. 2 and 3). However, plant-soil interactions do not affect the competition intensities $c_{\mathrm{A}}$ and $c_{\mathrm{B}}$, and hence, also not the stability of an internal equilibrium if it exists (Fig. 2).

While our analysis supports Bever's conclusion that negative feedback favors coexistence, we also demonstrated that the standard criterion for plant coexistence (the mutual invasibility of monocultures) is not a necessary condition for coexistence. As a consequence, 
plant coexistence under positive feedback is more likely than Bever's (2003) analysis suggests. Surely, in natural situations positive plant-soil feedback, for example, mediated by the presence of arbuscular mycorrhizal fungi is often associated with an increase in the dominance of a particular plant species and a reduction in diversity (Hartnett and Wilson 1999). Moreover, exotic plant species that escaped from species-specific soil pathogens may still profit from mutualistic root symbionts in their new range which favors their dominance (Callaway et al. 2004; Klironomos 2002). However, it has also been shown that positive plant-soil feedbacks can enhance plant coexistence. For example, the presence of arbuscular mycorrhizal fungi may enhance plant diversity in grasslands (Grime et al. 1987; van der Heijden et al. 1998b) by promoting seedling establishment and enhancing the competitive ability of subordinate plant species (Grime et al. 1987; van der Heijden et al. 2008).

Our results provide a mechanistic understanding of how negative plant-soil feedback can drive oscillations in plant species abundances. Oscillations could occur in situations where plants would otherwise competitively exclude each other, meaning that oscillations can enable coexistence. We also found, however, that oscillations along a heteroclinic orbit could occur in situations where plants would otherwise stably coexist, meaning that oscillations can also enable stochastic extinction. Therefore, negative plant-soil feedback does not necessarily enhance coexistence in all situations. Although many studies indicated that negative plant-soil feedback can be important driving plant dynamics (Olff et al. 2000) and ecological succession (De Deyn et al. 2003; van der Putten et al. 1993), there are no studies directly testing its impact on competitive oscillations. Yet, some of these empirical studies show that soil-borne organisms have the potential to decline the competitive strength of plants to such an extent that can be replaced by others (van der Putten and van der Stoel 1998; van der Putten et al. 1993).

We introduced two alternative interaction coefficients $J_{\mathrm{S}}$ and $H_{\mathrm{S}}$. These coefficients are more generally applicable to the current model, than Bever's $I_{\mathrm{S}}$. In the original plant-soil feedback model of Bever et al. (1997), plant abundances as well as soil communities are accounted in terms of proportions (not densities) and plant competition is "apparent" (sensu Holt 1977), being a by-product of the feedbacks. As a consequence, the original Bever et al. model (1997) has only two outcomes that can be predicted in terms of plant-soil feedback effects, i.e., the sign of $I_{\mathrm{S}}$. In contrast, the version of the Bever (2003) model that we studied here is a modification of the Lotka-Volterra model. This model has more degrees of freedom because plant densities can vary independently, and because this model contains additional parameters related to resource competition. Consequently, equilibrium stability and global dynamics cannot be fully accounted for by solely considering combinations of feedback effects $\left(I_{\mathrm{S}}, J_{\mathrm{S}}, H_{\mathrm{S}}\right)$, but must consider resource competition (parameters $c_{\mathrm{A}}$ and $c_{\mathrm{B}}$ ) as well. In this study, we have shown how Bever's coefficient $I_{\mathrm{S}}$ can be generalized to capture situations involving plant competition as well $\left(J_{\mathrm{S}}\right.$ or $\left.H_{\mathrm{S}}\right)$.

A major strength of the plant-soil feedback modeling approach introduced by Bever and colleagues is its close connection to experimental plant-soil feedback pot experiments (Bever 1994; Bever 1999; Bever et al. 1997). However, the assumption of exponential plant growth, made in the original version of the model, is problematic, even in shorter-term pot experiments. This may be one reason why the original model does not predict the outcome of such experiments very well (Kulmatiski et al. 2011). By linking plant-soil feedback to the classical Lotka-Volterra competition model, Bever (2003) included plant competition in his model. By providing the mathematical and graphical tools for analyzing this more complicated model, we hope to facilitate the extension of the plant-soil feedback approach to longer-term experiments and field situations.

Acknowledgments We thank Wim van der Putten, Han Olff, Johan van de Koppel, Peter de Ruiter, and one anonymous reviewer for comments on previous versions of the manuscript. TR was supported by a grant from the Netherlands Organization for Scientific Research (NWO).

Open Access This article is distributed under the terms of the Creative Commons Attribution License which permits any use, distribution, and reproduction in any medium, provided the original author(s) and the source are credited.

\section{Appendix A: Graphical analysis}

Figure 4 is useful for classifying and describing the main features of the dynamics in terms of the invasion conditions. For example in cases $1,2,11$, and 12, it is easy to see that only one species A or B always wins because only one of them is able to grow for any soil composition. In cases 4 and 14 , any resident species will be protected against invasion, and any equilibrium that may exist will be competitively unstable, for any soil composition.

However, cases 3, 5-10, 13, and 15-20 are more complicated. In these situations, it is sometimes useful to have a graphical representation in the familiar form of a phase space and nullclines. Although this is possible for a three-dimensional system like the Bever 
model, the following two-dimensional representation is more convenient:

\section{3 (positive feedback)}

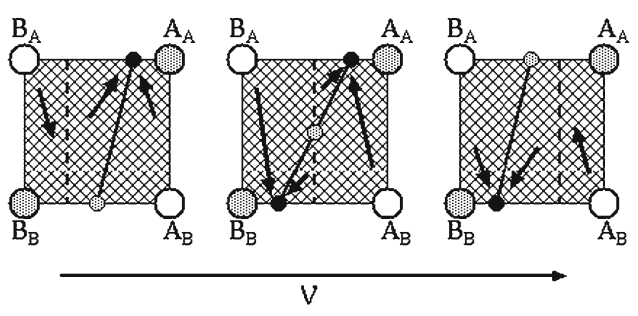

\section{3 (negative feedback)}
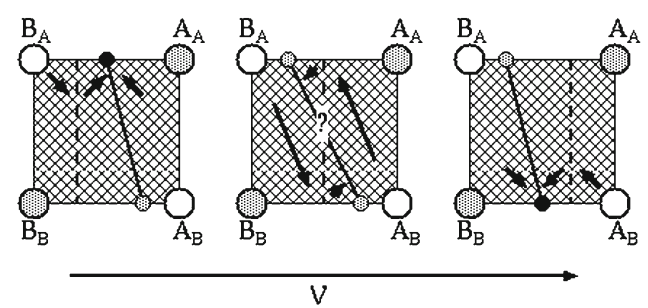

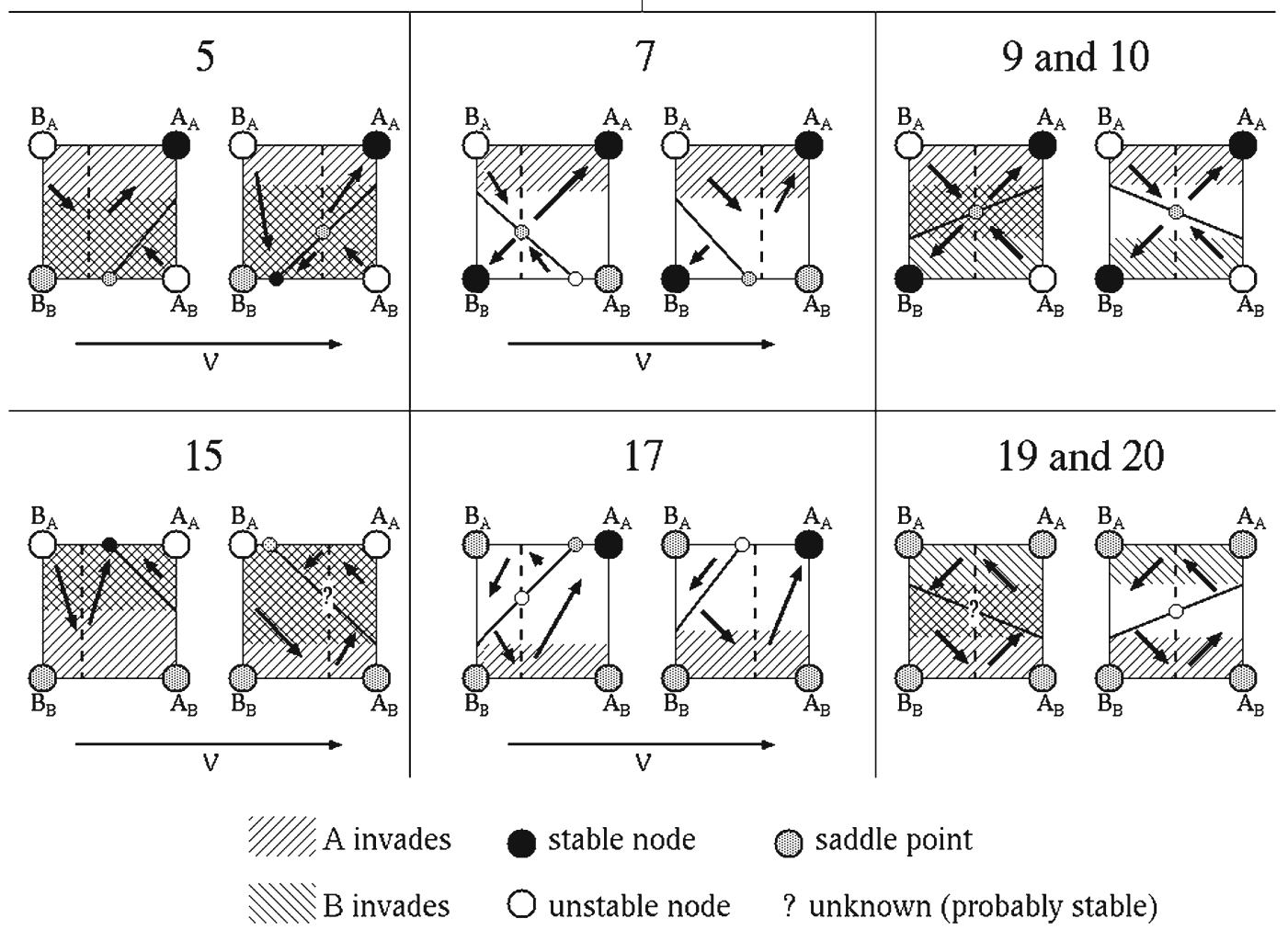

The phase space is constructed according to the following rules:

- The horizontal axis indicates the plant composition: plant $\mathrm{A}$ is dominant on the right, plant $\mathrm{B}$ on the left. The vertical axis indicates soil composition: A's soil biota dominates on the top, B's soil biota dominates on the bottom.

- The corners represent the plant monocultures, with $\mathbf{A}_{\mathbf{B}}$, $\mathbf{B}_{\mathbf{B}}$ corresponding to $S_{\mathrm{A}}=0$, and $\mathbf{A}_{\mathbf{A}}, \mathbf{B}_{\mathbf{A}}$ corresponding to $S_{\mathrm{A}}=1$. Depending on the competitive stability conditions $(7,8,11,12)$ and their soil stability, the corners are classified as stable, unstable, or saddle points.

- The invasion zones intersected by the feasibility arc become the domains of attraction in the phase plot, placed in the same order as they are encountered by traversing the arc from $S_{\mathrm{A}}=0$ to 1 (and using the same fill patterns).

- If the arc intersects zones III or IV, the corresponding domain of attraction in the phase plot is divided by a diagonal line. This line, representing coexistence equilibria, is the plant nullcline, i.e., a nullcline for the plant composition, not the plant densities. In the case of intersecting zone III, the plant composition moves towards the line (communities are competitively stable). In the case of intersection with zone IV, the plant composition moves away from the line (communities are competitively unstable).

- The plane is divided by vertical that represents the nontrivial soil nullcline: $S_{\mathrm{A}}$ increases at the right of the line 
$\left(N_{\mathrm{A}}>v N_{\mathrm{B}}\right.$ in Eq. 2), and decreases at the left $\left(N_{\mathrm{A}}<v N_{\mathrm{B}}\right.$ in Eq. 2). The smaller the $v$ the bigger the portion of the plane where $S_{\mathrm{A}}$ increases, and vice versa. The top $\left(S_{\mathrm{A}}=1\right)$ and the bottom $\left(S_{\mathrm{A}}=0\right)$ sides of the plane are trivial soil nullclines.

- A coexistence equilibrium corresponds to the intersection of the plant nullcline with a soil nullcline, trivial or not. For this reason, there can be border equilibria where $S_{\mathrm{A}}=0, S_{\mathrm{A}}=1$, or an internal equilibrium where $S_{\mathrm{A}}$ is intermediate. Depending on its location with respect to the attraction domains and the non-trivial plant nullcline, an equilibrium is declared stable, unstable or a saddle point.

Because of symmetry, we do not show cases $6,8,16$, and 18 because they are qualitatively equivalent to cases 5,7 , 15, and 17 (by swapping the "A" and "B" labels). Cases 3 and 13 are very similar in the stability of their monocultures, and because of having border equilibria. However, they display qualitatively different dynamics. Under net positive feedbacks (case 3 ) the system can display alternative stable states: coexistence with dominance of plant $\mathrm{A}$ and its soil community or coexistence with dominance by plant B and its soil community. On the other hand, under net negative feedbacks (case 13) there cannot be alternative stable states, and oscillations may develop (though we suspect they dampen out given the geometry of the nullclines).

The majority of cases under net positive feedback result in competitive exclusion. However, some can display alternative stable states, and coexistence depending on the initial conditions (5 and 6). On the other hand, the majority of scenarios under net negative feedback promote mutual invasion and coexistence (17 and 18 are the exceptions), including coexistence through oscillations (19 and 20).

\section{References}

Bever JD (1994) Feedback between plants and their soil communities in an old field community. Ecology 75:1965-1977. doi:10.2307/1941601

Bever JD (1999) Dynamics within mutualism and the maintenance of diversity: inference from a model of interguild frequency dependence. Ecol Lett 2:52-62. doi:10.1046/j.1461-0248.1999.21050.x

Bever JD (2003) Soil community feedback and the coexistence of competitors: conceptual frameworks and empirical tests. New Phytol 157:465-473. doi:10.1046/j.1469-8137.2003.00714.x

Bever JD, Westover KM, Antonovics J (1997) Incorporating the soil community into plant population dynamics: the utility of the feedback approach. J Ecol 85:561-573. doi:10.2307/ 2960528

Bolker BM, Pacala SW, Neuhauser C (2003) Spatial dynamics in model plant communities: What do we really know? Am Nat 162:135-148. doi:10.1086/376575

Bonanomi G, Giannino F, Mazzoleni S (2005) Negative plant-soil feedback and species coexistence. Oikos 111:311-321. doi:10.1111/j.0030-1299.2005.13975.x
Bradley DJ, Gilbert GS, Martiny JBH (2008) Pathogens promote plant diversity through a compensatory response. Ecol Lett 11:461469. doi:10.1111/j.1461-0248.2008.01162.x

Callaway RM, Thelen GC, Rodriguez A, Holben WE (2004) Soil biota and exotic plant invasion. Nature 427:731-733. doi:10.1038/ nature 02322

Case T (2000) An illustrated guide to theoretical ecology. University Press, Oxford

De Deyn GB, Raaijmakers CE, Zoomer HR, Berg MP, de Ruiter PC, Verhoef HA, Bezemer TM, van der Putten WH (2003) Soil invertebrate fauna enhances grassland succession and diversity. Nature 422:711-713. doi:10.1038/nature01548

Eppinga MB, Rietkerk M, Dekker SC, De Ruiter PC, van der Putten WH (2006) Accumulation of local pathogens: a new hypothesis to explain exotic plant invasions. Oikos 114:168-176. doi:10.1111/ j.2006.0030-1299.14625.x

Eppstein MJ, Molofsky J (2007) Invasiveness in plant communities with feedbacks. Ecol Lett 10:253-263. doi:10.1111/j.14610248.2007.01017.x

Eppstein MJ, Bever JD, Molofsky J (2006) Spatio-temporal community dynamics induced by frequency dependent interactions. Ecol Model 197:133-147

Gange AC, Brown VK, Sinclair GS (1993) Vesicular arbuscular mycorrhizal fungi-a determinant of plant community structure in early succession. Funct Ecol 7:616-622. doi:10.2307/ 2390139

Gilpin ME (1975) Limit cycles in competition communities. Am Nat 109:51-60

Grime JP, Mackey JML, Hillier SH, Read DJ (1987) Floristic diversity in a model system using experimental microcosms. Nature 328:420-422. doi: $10.1038 / 328420 \mathrm{a} 0$

Harper JL (1977) Plant population biology. Academic Press, New York

Hartnett DC, Wilson GWT (1999) Mycorrhizae influence plant community structure and diversity in tallgrass prairie. Ecology 80:1187-1195. doi:10.1890/0012-9658(1999) 080[1187: MIPCSA]2.0.CO;2

Holt RD (1977) Predation, apparent competition, and the structure of prey communities. Theor Popul Biol 12

Huisman J, Weissing FJ (2001) Biological conditions for oscillations and chaos generated by multispecies competition. Ecology 82:2682-2695. doi:10.1890/0012-9658(2001) 082[2682: BCFOAC]2.0.CO;2

Klironomos JN (2002) Feedback with soil biota contributes to plant rarity and invasiveness in communities. Nature 417:67-70. doi:10.1038/417067a

Klironomos JN (2003) Variation in plant response to native and exotic arbuscular mycorrhizal fungi. Ecology 84:2292-2301. doi: $10.1890 / 02-0413$

Kulmatiski A, Beard KH, Stevens JR, Cobbold SM (2008) Plant-soil feedbacks: a meta-analytical review. Ecol Lett 11:980-992. doi:10.1111/j.1461-0248.2008.01209.x

Kulmatiski A, Heavilin J, Beard KH (2011) Testing predictions of a three-species plant-soil feedback model. J Ecol 99:542-550. doi:10.1111/j.1365-2745.2010.01784.x

May RM, Leonard WJ (1975) Nonlinear aspects of competition between 3 species. SIAM J Appl Math 29:243-253

Mills KE, Bever JD (1998) Maintenance of diversity within plant communities: soil pathogens as agents of negative feedback. Ecology 79:1595-1601. doi:10.1007/s004420050823

Olff H, Hoorens B, de Goede RGM, van der Putten WH, Gleichman JM (2000) Small-scale shifting mosaics of two dominant grassland species: the possible role of soil-borne pathogens. Oecologia 125:45-54. doi:10.1007/PL00008890

Revilla T (2009) Multispecies resource competition. PhD dissertation. University of Groningen, The Netherlands 
Revilla T, Weissing FJ (2008) Nonequilibrium coexistence in a competition model with nutrient storage. Ecology 89:865-877. doi: org/10.1890/07-1103.1

Reynolds HL, Packer A, Bever JD, Clay K (2003) Grassroots ecology: plant-microbe-soil interactions as drivers of plant community structure and dynamics. Ecology 84:2281-2291. doi:10.1890/02-0298

Tilman D (1988) Plant strategies and the dynamics and structure of plant communities. Princeton University Press, NJ

Umbanhowar J, McCann K (2005) Simple rules for the coexistence and competitive dominance of plants mediated by mycorrhizal fungi. Ecol Lett 8:247-252. doi:10.1111/j.14610248.2004.00714.x

van der Heijden MGA, Boller T, Wiemken A, Sanders IR (1998a) Different arbuscular mycorrhizal fungal species are potential determinants of plant community structure. Ecology 79:2082-2091. doi:10.1890/0012-9658(1998) 079[2082:DAMFSA]2.0.CO;2

van der Heijden MGA, Klironomos JN, Ursic M, Moutoglis P, Streitwolf-Engel R, Boller T, Wiemken A, Sanders IR (1998b) Mycorrhizal fungal diversity determines plant biodiversity, ecosystem variability and productivity. Nature 396:69-72. doi:10.1038/23932

van der Heijden MGA, Wiemken A, Sanders IR (2003) Different arbuscular mycorrhizal fungi alter coexistence and resource distribution between co-occurring plant. New Phytol 157:569578. doi:10.1046/j.1469-8137.2003.00688.x

van der Heijden MGA, Bardgett RD, van Straalen NM (2008) The unseen majority: soil microbes as drivers of plant diversity and productivity in terrestrial ecosystems. Ecol Lett 11:296-310. doi:10.1111/j.1461-0248.2007.01139.x

van der Putten WH, van der Stoel CD (1998) Plant parasitic nematodes and spatio-temporal variation in natural vegetation. Appl Soil Ecol 10:253-262. doi:10.1016/S0929-1393(98)00124-3

van der Putten WH, van Dijk C, Peters BAM (1993) Plant-specific soil-borne diseases contribute to succession in foredune vegetation. Nature 362:53-56. doi:10.1038/362053a0

van der Putten WH, Vet LEM, Harvey JA, Wäckers FL (2001) Linking above- and belowground multitrophic interactions of plants, herbivores, pathogens, and their antagonists. Trends in Ecol Evol 16:547-554. doi:10.1016/S0169-5347(01)02265-0

van der Putten WH, Bardgett RD et al (2009) Empirical and theoretical challenges in aboveground-belowground ecology. Oecologia 161:1-14. doi:10.1007/s00442-009-1351-8

Wardle DA, Bardgett RD, Klironomos JN, Setälä H, van der Putten WH, Wall DH (2004) Ecological linkages between aboveground and belowground biota. Science 304:1629-1633. doi:10.1126/ science. 1094875 